\title{
BIFURCATION PHENOMENA IN THE SHORT TAYLOR-COUETTE CAVITY WITH THE ASYMMETRIC END-WALLS AT LOW Re
}

\author{
E. Tuliszka-Sznitko \\ Poznan University of Technology, Piotrowo 3, 60-965 Poznan, Poland
}

\begin{abstract}
The paper presents the results of the numerical investigations (DNS) of unsteady phenomena observed in the short Taylor-Couette configurations $\left(\Gamma=H /\left(R_{2}-R_{1}\right)=2.6-4.0\right)$ of different radii ratios $\eta=R_{1} / R_{2}=0.25-0.6$ with the asymmetric end-wall boundary conditions. The computations are performed at low Reynolds numbers i.e. $\operatorname{Re}=\Omega \mathrm{R}_{1}\left(\mathrm{R}_{2}-\mathrm{R}_{1}\right) / v=100-200$. In such configurations many interesting bifurcation phenomena occur: homoclinic, heteroclinic and doubling period. The paper is thought as complementary to Mullin, Blohm [1] where the analysis is limited to $\eta=0.5$. The present DNS results confirm that for $\eta=0.5$ the flow dynamics is organized by a pair of codimension-2 bifurcation points. The DNS study has allowed for the determination of the neutral curves in the 3D parameter space $(\operatorname{Re}, \Gamma, \eta)$ and the detailed analysis of the modulated rotating waves for different $\eta$. The study has shown that the MRW behavior depends strongly on $\eta$ and Re. These results are presented in the light of Lopez et al. [2] observations.
\end{abstract}

KEY WORDS: Taylor-Couette flow, bifurcation phenomena, modulated rotating wave, DNS

\section{INTRODUCTION}

Mullin and Blohm [1] have studied the bifurcation phenomena in the short Taylor-Couette configurations of aspect ratios between $\Gamma=H /\left(R_{2}-R_{1}\right)=2.4-3.8$ and radii ratio $\eta=0.5$ with the asymmetric end-wall boundary conditions numerically using the ENTWIFE solver and experimentally using the LDV method. They have shown that the 3-cell structure (created with the increase of Re) collapses to the 1-cell structure via a saddlenode bifurcation at the critical value of Re. The decrease of Re leads to the reformation of the 1-cell structure to 3-cell structure. The paths of saddle-nodes meet at the cusp point which is connected with the existence of the local time dependent area. Mullin and Blohm [1] have found that the fluid flow dynamics in this area is organized by a pair of codimension-2 bifurcation points i.e. the cusp point and the double Hopf bifurcation point where paths of two Hopf bifurcations intersect. The Hopf bifurcations are supercritical and symmetry breaking which result in the appearance of two $3 \mathrm{D}$ time-periodic waves $\mathrm{RW}_{1}$ and $\mathrm{RW}_{2}$ revealed numerically by Lopez et al. [2]. Lopez et al. [2] have studied in detail the flow dynamics in the vicinity of the cusp point where the modulated rotating wave (MRW) occurs (the $\mathrm{RW}_{1}$ wave becomes unstable via the supercritical Neimark-Sacker bifurcation). They have performed one parameter analysis for $\eta=0.5, \operatorname{Re}=120$ and have shown that with the increase of $\Gamma$ the MRW period increases (in the limit $\mathrm{T}_{\mathrm{MRW}} \rightarrow \infty$ ). Lopez et al. [2] have found that the modulated rotating wave undergoes some saddle-node bifurcations to disappear after homoclinic/heteroclinic collision. The bifurcation phenomena in the short Taylor-Couette configurations have been investigated also by Pfister [3], Mullin [4], Balackburn, Lopez [5], Lorenzen et al. [6], Mullin et al. [7], among others. Pfister [3] has shown experimentally that in the Taylor-Couette flow of very small aspect ratio $\Gamma=0.36, \eta=0.5$ the presence of MRW gives rise to the period doubling state. 
The main focus here is the effect of radii ratio $\eta$ on the bifurcation phenomena described by Mullin, Blohm [1] and Lopez et al. [2] for $\eta=0.5$. To answer this question the systematic 3D DNS computations are performed in the short Taylor-Couette cavities of different $\eta$.

\section{NUMERICAL METHODS}

In DNS the incompressible flow is described by the Navier-Stokes equation written in a cylindrical coordinate system. The flow is confined in an annulus of the inner cylinder radius $\mathrm{R}_{1}$ and the outer cylinder radius $R_{2}$. The flow is driven by the bottom rotating disk and the inner cylinder which rotate at $\Omega[\mathrm{rad} / \mathrm{s}]$. The length is normalized by the gap between cylinders $R_{2}-R_{1}$, time is normalized by the diffusive time scale $\left(R_{2}-\right.$ $\left.\mathrm{R}_{1}\right)^{2} / v$. The spatial scheme is based on a pseudo-spectral Chebyshev-Fourier-Galerkin collocation approximation. In time approximation a second-order semi-implicit scheme is used. More information can be found in $[8,9]$. The saddle-node bifurcation paths are determined using the finite elements ENTWIFE code which delivers the steady and axisymmetric solutions, Mullin, Blohm [1].

\section{THE RESULTS}

The exemplary saddle-node lines obtained for different $\eta$ have been presented in [10]. The ENTWIFE results show that for all considered radii ratios $(\eta=0.25-0.6)$ the sequence of observed phenomena in the exchange process between the 3-cell structure and the 1-cell structure is qualitatively the same in spite of the variety of considered configurations. The present DNS results obtained for $\eta=0.5$ reproduce the experimental and numerical data published by Mullin, Blohm [1] and confirm that for $\eta=0.5$ the flow dynamics is organized by a pair of codimension-2 bifurcations. In all considered flow cases only the single spiral $\mathrm{RW}_{1}$ and the double spiral $\mathrm{RW}_{2}$ waves are observed as it has been described for $\eta=0.5$ in [2]. Amplitudes and frequencies of $\mathrm{RW}_{1}$ and $\mathrm{RW}_{2}$ are obtained from the radial velocity component time series determined in the middle point of cavity. The squared amplitudes of the radial velocity component $(2 \mathrm{~A})^{2}$ of both waves $\mathrm{RW}_{1}$ and $\mathrm{RW}_{2}$ grow and decay linearly with the increase and decrease of Re. This behavior is consistent with the Hopf bifurcation theory. The neutral curves are determined for all considered $\eta$. In the next step, the behavior of the modulated rotating wave is investigated. The most important information on the MRW wave Lopez et al. [2] have obtained from the one-parameter analysis (the period $\mathrm{T}_{\mathrm{MRW}}$ is studied as a function of $\Gamma$, Re fixed). The authors have revealed the existence of two critical values of $\Gamma$ for which $T_{M R W} \rightarrow \infty$ between which only the stable A3 solution exists. Lopez et al. [2] have concluded that such behavior of $\mathrm{T}_{\mathrm{MRW}}$ is typical when the cycle approaches and becomes homoclinic at $\Gamma_{\mathrm{c}}$. After verifying the present computations by comparison of the modulated period $\mathrm{T}_{\mathrm{MRW}}$ obtained for $\eta=0.5(\mathrm{Re}=120)$ and selected $\Gamma$ with the Lopez et al. [2] results, the one-parameter paths are determined for $\eta=0.3,0.375,0.42,0.475,0.6$ and for different Re. The results show that for radii ratio from the range of $\eta=0.375-0.42$ the strong doubling bifurcation process is observed whereas for larger $\eta$ the singled period bifurcation dominates.

The one-parameter path obtained in the present study for $\mathrm{Re}=119.7, \eta=0.475$ and this obtained by Lopez et al. [2] for $\eta=0.5, \operatorname{Re}=120$ are, in fact, shifted by approximately 0.0713 (the critical value of $\Gamma$ obtained for $\eta=0.5$ equals 2.9691 , whereas, for $\eta=0.475$ equals 3.04041 ). The time series obtained for 100 consecutive azimuthal sections show that the flow of $\Gamma=3.0404$ is $2 \mathrm{D}$ in almost the whole $\mathrm{T}_{\mathrm{MRW}}$. The flow is strongly $3 \mathrm{D}$ only in the narrow areas where the modal energy $u_{1} \cdot u_{1}^{*} / 2$ reaches a large values $(m=1$ Fourier component). In the present investigation the similar bifurcation phenomena are observed for $\eta=0.6-0.45$. The similarity of the one-parameter paths and the phase portraits (Fig.2) obtained for these flow examples indicates that the Lopez's et al. [2] scenario of bifurcation phenomena, which includes the Hopf bifurcation, Neimark-Sacker bifurcation and heteroclinic collision, is valid for the wider range of $\eta$. However, with the decrease of $\eta$ the time series become more complex. Again, the $T_{M R W}$ period increases with the increase of $\Gamma$ but for the flow case of $\eta=0.42, \operatorname{Re}=119.364$ this process is very rapid (Fig. 1a) and rather similar to the doubling process 
described by Pfister [3] than to this obtained for $\eta=0.475$ and 0.5 . The time series obtained for 100 azimuthal sections $(\eta=0.42, \operatorname{Re}=119.364, \Gamma=3.169$, Fig. $1 b)$ show strong three-dimensionality of this flow case (every eight hundredth value from the time series is used to create this figure). The phase portrait is presented in Fig.2b (the phase portraits become more and more complex with the increase of $\Gamma$ ). For the flow case of $\eta=0.42$ the doubling period bifurcation is observed in the narrow range of Reynolds number $\operatorname{Re}=117-120$. For higher Re the singled period bifurcation dominates. The most interesting flow dynamics is observed for $\eta=0.375$ where the rapid (doubling) increase of $T_{\text {MRW }}$ occurs at the relatively wide range of Re. The time series and the phase portraits are similar to these observed for $\operatorname{Re}=119.364, \eta=0.42$. In the flow case of $\eta=0.3$ the MRW wave occurs at the similar range of Re as for $\eta=0.375$ but the increase of $T_{M R W}$ with the increase of $\Gamma$ is smoother.
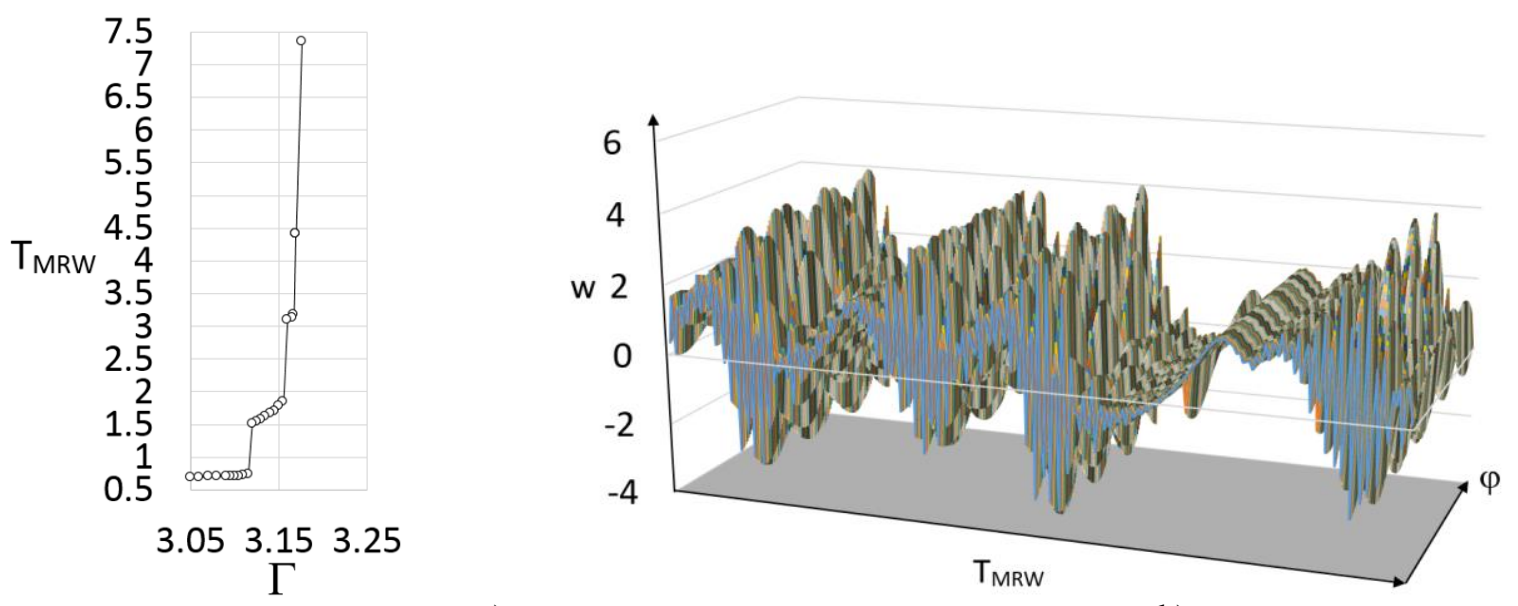

a)

b)

Fig. 1 a) The $T_{M R W}$ period as a function of aspect ratio, $R e=119.364$, b) the time series obtained in 100 azimuthal sections, $\operatorname{Re}=119.364, \Gamma=3.169, \eta=0.42$.

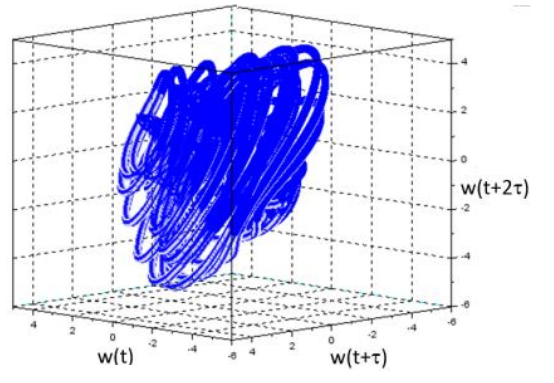

a)

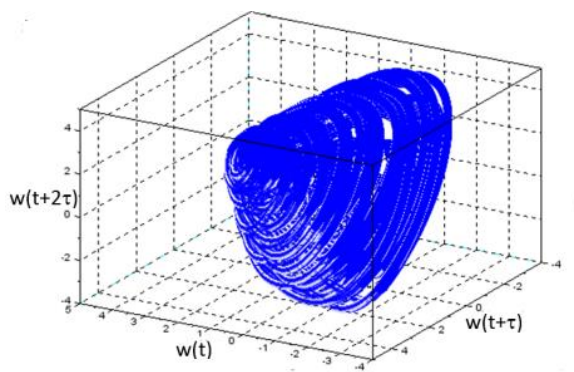

b)

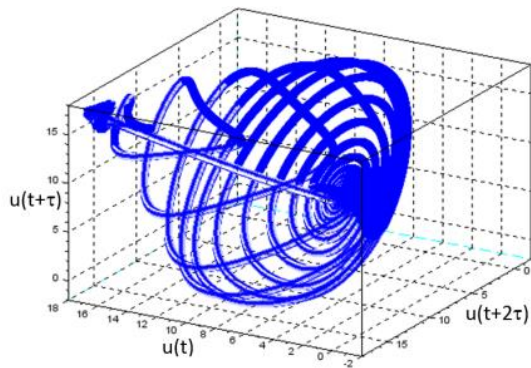

c)

Fig. 2 The phase portraits obtained using the delay method, delay $\tau=0.02$, a) $\eta=0.375, \operatorname{Re}=121.78, \Gamma=3.3255$, b) $\eta=0.42, \operatorname{Re}=119.364, \Gamma=3.169$, c) $\eta=0.475, \operatorname{Re}=119.7, \Gamma=3.0404$.

\section{CONCLUSIONS}

The investigations have been performed in the cavities of radii ratios from the range of $\eta=0.25-0.6$ and the $\Gamma$ values have been selected in such a way that only the 1- and 3-cell structures occur. The results are discussed in the light of Mullin, Blohm [1] and Lopez et al. [2] findings, $\eta=0.5$. The present DNS results have confirmed that for $\eta=0.5$ the flow dynamics is organized by a pair of codimension- 2 bifurcations and have 
reproduced the curves of secondary Hopf bifurcations. The DNS results have shown that the squared amplitudes $(2 \mathrm{~A})^{2}$ of the radial velocity component of the $\mathrm{RW}_{1}$ and $\mathrm{RW}_{2}$ waves grow and decay linearly with the increase and decrease of $\operatorname{Re}$ for all $\eta$. The $(2 \mathrm{~A})^{2}$ profiles have enabled for the determination of the unsteady areas in the $3 \mathrm{D}(\operatorname{Re}, \Gamma, \eta)$ parameter space.

The present DNS study has shown that the MRW behavior depends strongly on $\eta$ and Re. For $\eta=0.45-0.6$ the similar bifurcation processes to those described in [2] for $\eta=0.5, \mathrm{Re}=120$ (homoclinic/heteroclinic collision when $\mathrm{T}_{\mathrm{MRW}} \rightarrow \infty$ ) have been observed. However, for the flow case of $\eta=0.42$ and low Reynolds number $\mathrm{Re}=119.364$ the doubled period cascade has been observed. The phase portraits have become more and more complex with the increase of $\Gamma$. This rapid increase of $T_{M R W}$ has disappeared for Reynolds number slightly higher than 120.6 (the MRW wave becomes singled period). For the flow case of $\eta=0.375$ the doubled period occurs in larger range of Re in comparison with $\eta=0.42$. The collision line " $C$ " has been determined for $\eta=0.375,0.475$ and 0,6 and for different Re, and compared with the results of Lopez et al. [2]. The modal energy as a function of time is analyzed. For the flow case of $\eta=0.3$ the changes of $T_{M R w}$ as a function of $\Gamma$ are more mild.

\section{ACKNOWLEDGMENT}

The author is grateful to Prof. Tom Mullin from Oxford University for inspiration, fruitful discussions and research help. The DNS computations have been performed in Poznan Supercomputing and Networking Center, which is gratefully acknowledged.

\section{NOMENCLATURE}

$\begin{array}{llllll}\mathrm{H} & \text { height of cavity } & (\mathrm{m}) & \eta & \text { radii ratio } & (-) \\ \mathrm{R}_{1}, \mathrm{R}_{2} & \text { radii of cylinders } & (\mathrm{m}) & \Gamma & \text { aspect ratio } & (-) \\ \mathrm{Re} & \text { Reynolds number } & (-) & v & \text { kinematic viscosity } & \left(\mathrm{m}^{2} / \mathrm{s}\right) \\ \mathrm{T}_{\mathrm{MRW}} & \text { period of MRW } & (-) & \Omega & \text { rotation } & \left(\mathrm{rad} \mathrm{s}^{-1}\right) \\ \mathrm{u}, \mathrm{W} & \mathrm{r}, \mathrm{z} \text { velocity components } & (-) & & & \end{array}$

\section{REFERENCES}

[1] Mullin, T. and Blohm, C., "Bifurcation phenomena in a Taylor-Couette flow with asymmetric boundary conditions", Phys. Fluids, 13, 1, pp. 136-140, (2001).

[2] Lopez, J.M., Marques, F., Shen, J., "Complex dynamics in a short annular container with rotating bottom and inner cylinder", J. Fluid Mech., 501, pp. 327-354, (2004).

[3] Pfister, G., "The deterministic chaos in rotational Taylor-Couette flow", In G.E.A. Meier and F., Obermeier, Eds., Flow of Real Fluid, Lecture Notes in Physics, 235, Berlin Springer, pp. 199-210, (1985).

[4] Mullin, T., "Finite-Dimensional Dynamics in Taylor-Couette Flow", IMA J. App. Math., 46, pp. 109-119, (1991).

[5] Balackburn, H.M. and Lopez, J.M., "Modulated rotating waves in an enclosed swirling flow", J. Fluid Mech., 465, pp. 33-58, (2002).

[6] Lorenzen, A. and Mullin, T., "Anomalous modes and finite-length effects in Taylor-Couette flow", Phys. Rev. A, 31, p. 3463, (1985).

[7] Mullin, T., Tavener, S., Cliffe, K., “An Experimental and Numerical Study of a Codimension-2 Bifurcation in a Rotating Annulus”, Europhysics Letters, 8 (3), pp. 251-256, (1989).

[8] Serre, E. and Pulicani, J.P., "A three-dimensional pseudospectral method for rotating flows in a cylinder", J. Computers and Fluids, 30, 4, 491-519, (2001).

[9] Tuliszka-Sznitko, E., Zielinski, A., Majchrowski, W., "LES of the non-isothermal transitional flow in rotating cavity", Int. J. Heat and Fluid Flow, 30, 3, pp. 534-548, (2009).

[10] Tuliszka-Sznitko, E. and Mullin, T., "Numerical investigation of bifurcation phenomena in Taylor-Couette flow with the asymmetric end-wall boundary conditions", Book of Abstracts of 12th European Turbulence Conference, Stockholm, (2017). 